\title{
Control Algorithm and Simulation Research of Electric Boiler System
}

\author{
Ying Liu ${ }^{1, a}$ and Guiling Fan, b \\ ${ }^{1,2}$ Xijing University, Shaanxi Xi'an, 710123, China \\ a15905721@qq.com, b76861639@qq.com
}

Keywords: Control strategy; PID control; SMITH algorithm; DAHLIN control; Fuzzy control

\begin{abstract}
This paper studies and designs a temperature control system based on single-chip microcomputer as the core of the intelligent electric heating boiler, and the fuzzy control algorithm is adopted in the control strategy. PID control, SMITH algorithm, DAHLIN control and fuzzy control method are commonly used. The advantages and disadvantages of these methods are compared with the traditional algorithms, and the simulation results show that the control system has good control effect and can meet the practical needs.
\end{abstract}

\section{Introduction}

Electric boiler is a device that converts electric energy into heat energy, producing steam or hot water. It can meet the needs of high rise building, commercial, small industrial and mining enterprises, automobile, shipbuilding industry and other fields. Compared with other fuel boilers, electric heating boiler has the characteristics of high efficiency, high heat transfer coefficient, high heat transfer coefficient, and high thermal efficiency of 97\%. 20 years since the beginning of the application of electric heating boiler, domestic since the late 70's also carried out the application of electric boiler. At present, in the process of industrial production in our country, electric boiler has been widely used.

\section{Font and Type Style}

Temperature control of electric heating boiler is mainly controlled by PID. The control effect of PID is related to the selection of control parameters, and the tuning of PID parameters is a very complicated work. Although the tuning and optimization of PID parameters is a lot of methods, the traditional non intelligent setting method, such as Zieger-Nichols method, is clearly an empirical method and is not the optimal solution, and cannot obtain the ideal control effect. At present, the intelligent setting method such as fuzzy PID, neural network PID can better realize the optimization of PID control parameters, but it needs to be adjusted online, the calculation is large, the burden of the controller is very heavy. It is difficult to establish accurate mathematical model, and it is very difficult to establish a precise mathematical model. It is very difficult to establish a precise mathematical model. Nowadays, fuzzy control has been applied in many fields. Because the fuzzy control is mainly to imitate the human's control experience and not depend on the model of the control object, so fuzzy control can reflect the people's control behavior.

System hardware is composed of power circuit, temperature detection circuit, analog interface circuit, microcontroller system, man-machine interface and RS-485 bus interface, Principle diagram shown in Fig. 1. 


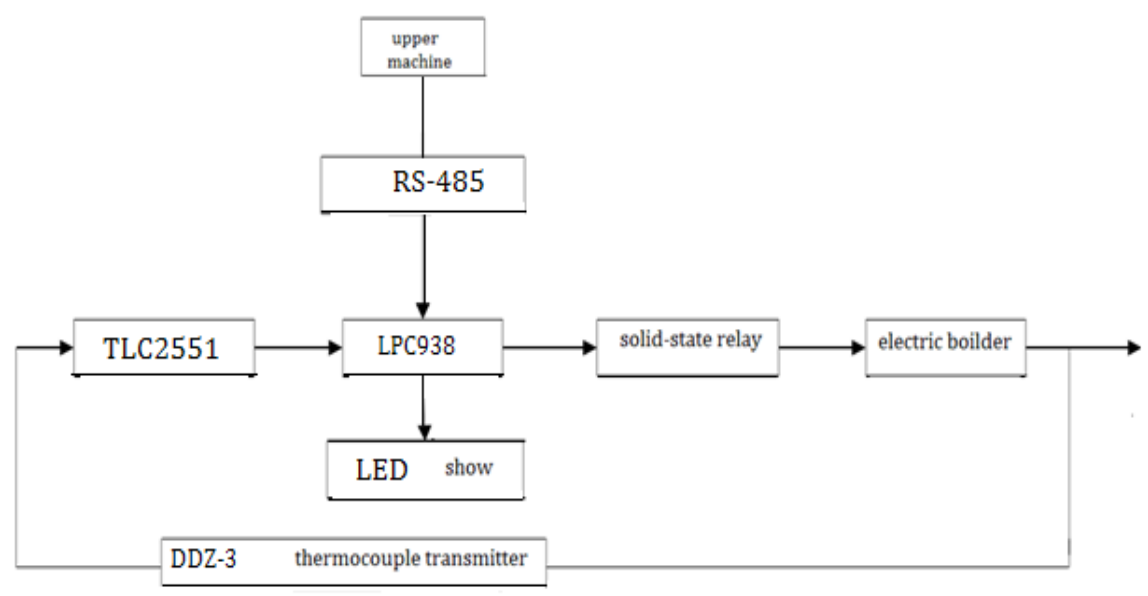

Figure 1. Hardware structure diagram of temperature control system

Working principle: by the integrated thermocouple transmitter to detect the system temperature, and complete the signal standardization, change function. The single chip microcomputer performs the function of fuzzy control, and is controlled by the solid state relay to control the power supply of the heater and the fault, so as to achieve the purpose of controlling the temperature. Through the RS-485 to complete the data communication between P89LPC938 and PC, the function of remote monitoring is realized.

The input voltage is $\mathrm{AC} 220 \mathrm{~V}$ and the output of the transformer is $9.5 \mathrm{~V}$, and the output is rectified. Rectification can be carried out through four diodes for full wave rectification, and also can be carried out using integrated rectifier. The back of the capacitor C19, C20 filter capacitor for filtering, and pay attention to the electrolytic capacitor should have a certain margin, otherwise it cannot play a very good filtering effect. The capacitor used in this circuit is $470 \mathrm{~F}$, and the voltage is $25 \mathrm{~V}$. The output level of the 78L05 is connected with two filter capacitor, which is used to reduce the influence and filtering of the system caused by the power fluctuation. Do not need to use large capacity electrolytic capacitor, capacity of $100 \mathrm{~F} 25 \mathrm{~V}$, and then access to $0.1 \mathrm{~F}$ capacitor, it can be reduced because of the influence of power fluctuations and filter to ripple, very good to improve the load transient response. However, a disadvantage is that once the 78L05 input is short circuit, the output end of a large capacitor on the storage of electric charge, will be through the integrated voltage regulator internal discharge, may cause damage to the internal circuit, so in the meantime cross a diode, to discharge the discharge path, the integrated voltage regulator has played a role in the diversion.

\section{PID Controller and Simulation}

PID Algorithm. PID is a negative feedback control, with the set of the control target value and the output feedback value of the controlled object, the difference of the proportion, differential, integral, used to control the controlled object. Structure as shown in Fig. 2:

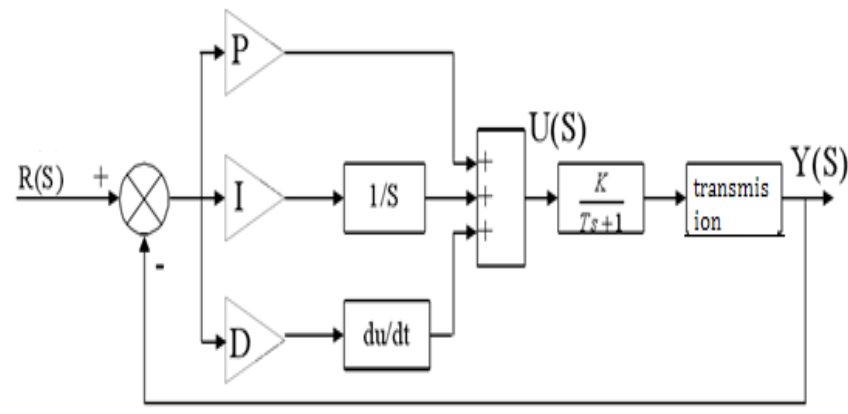

Figure 2. PID controller structure diagram 
PID control rule:

$$
u=\frac{1}{\delta}\left(e+\frac{1}{T_{I}} \int_{0}^{t} e d t+T_{D} \frac{d e}{d t}\right)
$$

The changes of DT, IT and $\delta$ have a great influence on the control effect. The bigger the difference is, the smaller the proportion of the adjustment of the proportion of the residuals is, from this point, the smaller the residual error is. However, the open loop gain of the control system is increased, which may cause the system to be unstable, and the system must be stable, so the setting of the proportional band must ensure a certain degree of stability margin. The bigger the IT is, the smaller the integral speed is, the more the integral function is. IT is faster and faster, and the integral speed can decrease the stability of control system, and the DT of the system can improve the dynamic performance of the system, which can speed up the system response, increase the system stability, but the DT is too large, and the anti-disturbance ability of the system is weakened. PID controller, the IT, DT, if appropriate, they can play their strengths, and thus better control system, otherwise, not only cannot play a variety of regulatory role, but counterproductive.

Simulation of PID Controller. System simulation is based on the mathematical model of the system, with a computer as a tool for the experimental study of the system. System simulation is based on the mathematical model of real system simulation model, the design, analysis, research, to obtain the quantitative relationship between the real system, the real system to deepen understanding and understanding, for system design, commissioning or management to provide the required information, data or information. The mathematical model of the system is deduced:

$$
G(s)=\frac{127}{292 s+1} e^{-15 s}
$$

For the PID controller parameters of the system, the Ziegler-Nichols tuning method, the improved Ziegler-Nichols and Cohen-Coon method are adopted respectively. When the temperature is $1000 \mathrm{C}$, the simulation results are shown in figure 3 :

The PID parameters are: $p_{k}=0.184, T_{i}=30, T_{d}=7.5$

The PID parameters are determined by using the modified Ziegler-Nichols method:

$\mathrm{p}_{\mathrm{k}}=0.184, \mathrm{~T}_{\mathrm{i}}=30, \mathrm{~T}_{\mathrm{d}}=7.5, \beta=0.672$

The PID parameters are determined by Cohen-Coon method:

$\mathrm{P}_{\mathrm{k}}=0.206, \mathrm{~T}_{\mathrm{i}}=71.928, \mathrm{~T}_{\mathrm{d}}=5.41$

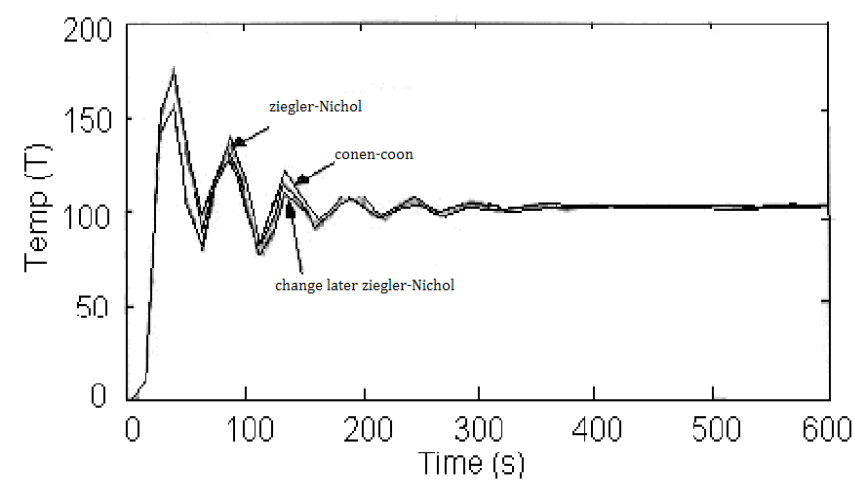

Figure 3. Simulation of PID control effect of different setting method

As shown in Fig.3, for the system, the overshoot of the curve obtained by Cohen-Coon tuning method is less than that of the Ziegler-Nichol tuning method. But the stability is slightly worse than that of Ziegler-Nichol. 


\section{SMITH Prediction Algorithm and Simulation}

SMITH Prediction Algorithm. Smith predictor compensation controller is a control algorithm based on the model, which can make the system with large pure delay has better control performance. Its characteristics is to pre estimate the dynamic characteristics of the process under the perturbation, and a compensation from the estimator, trying to delay tau variable advance reflect to the control volume, the advance control action, thus significantly decreases overshoot and accelerate the adjustment process. Smith predictor is one of the effective methods to overcome the effect of pure delay. Based on the conventional correction link, the Smith predictor is introduced. The control structure is shown in Fig.4, and the block diagram of the Smith pre estimation control in the dotted line. The closed-loop transfer function of the system is:

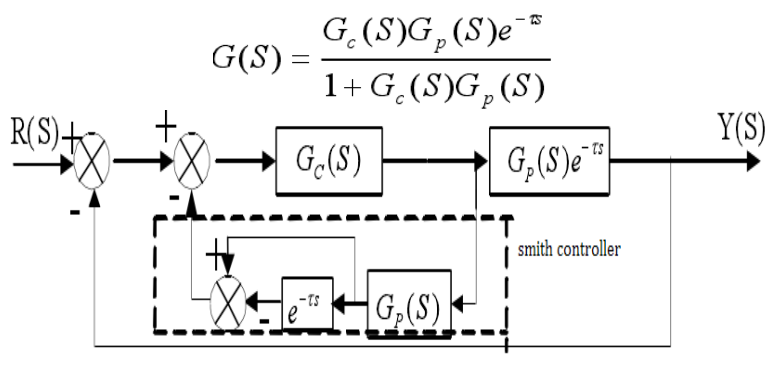

Figure 4. Smith prediction algorithm

SMITH Prediction Algorithm Simulation. For the system of the temperature object using Smith prediction algorithm, when the model is completely accurate, the controller GC1 (S) according to the PID controller parameters tuning method Cohen-Coon. Get Matlab simulation Fig. 5:

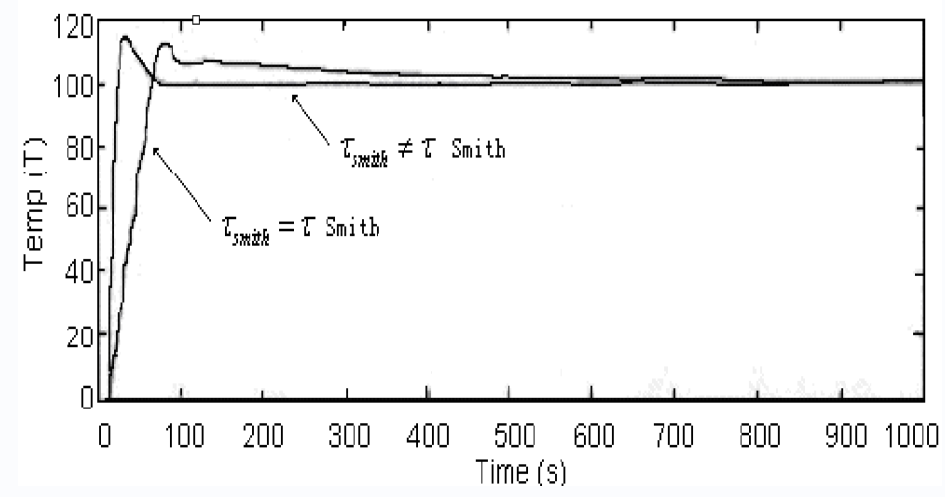

Figure 5. Smith prediction algorithm simulation

\section{DAHLIN Algorithm and Simulation}

DAHLIN Algorithm. Industrial thermal process contains a pure lag phase, easy to cause the system to overshoot, and even oscillation. But the stability of the system and the requirements of the smaller overshoot are the main parameters of the system. IBM Dahlin for this request to propose a control algorithm in the $\mathrm{Z}$ domain. Its structure is shown in Fig. 6:

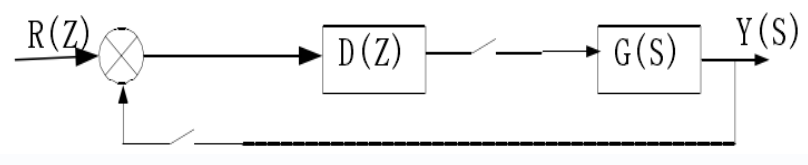

Figure 6. Dahlin algorithm structure diagram 
Dahlin algorithm design goal is to make the closed-loop system desired transfer function phi (s) is equivalent to a delay links and an inertia link connected in series, namely:

$$
\Phi(S)=\frac{1}{1+T_{\tau}(S)} e^{-\varpi}
$$

DAHLIN Algorithm Simulation. Dahlin algorithm is used to control the temperature of the system, if the gain does not increase, then the time temperature curve should not exceed. The simulation results are shown in Fig. 7:

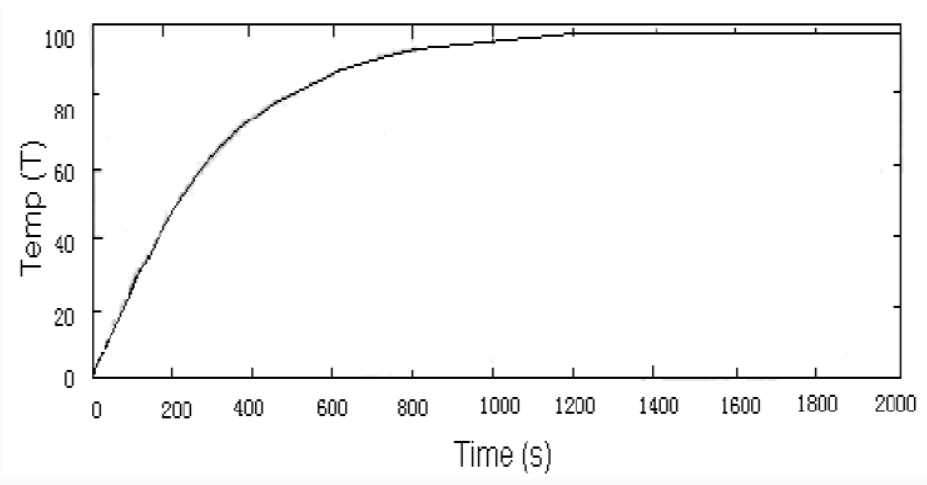

Figure 7. Dahlin algorithm simulation

\section{Summary}

In summary, the control system has obvious pure delay and time constant, in order to meet the conventional heating, heat preservation curve of the condition, not only requires its good stability, does not produce over tone, but also requires tracking performance and anti-jamming performance. It is clear that the conventional PID control, Smith budget method cannot meet the performance requirements; Dahlin controller is an effective discrete control algorithm, it is easy to understand the advantages of the controller design process, control system has good robustness, does not produce a super tone, and strong anti-interference ability. So it can be applied to the control of the pure delay process, but its fast tracking performance is poor, and the FUZZY control is the opposite, the time is short, fast tracking performance is good. Therefore, the fuzzy control method is used in this system.

\section{References}

[1] Ge M, Chiu M S, Wang Q G. Robust PID controller design via LMI approach. Journal of process control, 2012, (12): 3-13.

[2] Lee, Kyung Chang Lee, Suk. Performance evaluation of switched Ethernet for networked control systems IECON Proceedings. Industrial Electro-nics Conference,2012,(4):3170-3175

[3] Bristol E H. Pattern recognition An alternative to parameter identification in adaptive control. Automatica,2010,(13):197-202

[4] R Layne et al. Fuzzy Modle Reference Learning Control for Cargo Ship Steering. IEEE Contr Syst,2010,13(5):22-24

[5] E cox. Adaptive Fuzzy System. IEEE Spectrum, 2010,30(2) : 27-31

[6] B Kosko, S G Kong. Adaptive Fuzzy Systems for Backing up a Track-and-trailer. IEEE Trans Neural Networks, 2010,3(2):211-233

[7] Li, Mei-Jun. Design of intelligent PROFIBUS-DP slaver. Control and Instruments in Chemical Industry, 2013, 31(3): 33-35

[8] Forouzan, B.A.TCP/IP Protocol Suite. McGraw-Hill, 2000, (3):1444-1447 